\title{
A Sandwich-Structured Piezoresistive Sensor with Electrospun Nanofiber Mats as Supporting, Sensing, and Packaging Layers
}

\author{
Zicong Zhao ${ }^{1,2}$, Bintian Li ${ }^{1,2}$, Liqun Xu ${ }^{1,2}$, Yan Qiao ${ }^{1,2}$, Feng Wang ${ }^{3,4}\left(\mathbb{D}\right.$, Qingyou Xia ${ }^{3,4}$ (1) and \\ Zhisong Lu 1,2,*(D) \\ 1 Chongqing Key Laboratory for Advanced Materials \& Technologies of Clean Energies, Southwest University, \\ 1 Tiansheng Road, Chongqing 400715, China; zhaozc1993@163.com (Z.Z.); libint0506@163.com (B.L.); \\ xulq@swu.edu.cn (L.X.); Yanqiao@swu.edu.cn (Y.Q.) \\ 2 Institute for Clean Energy \& Advanced Materials, Faculty of Materials \& Energy, Southwest University, \\ 1 Tiansheng Road, Chongqing 400715, China \\ 3 State Key Laboratory of Silkworm Genome Biology, Southwest University, Chongqing 400715, China; \\ wangf1986@swu.edu.cn (F.W.); xiaqy@swu.edu.cn (Q.X.) \\ 4 Chongqing Engineering and Technology Research Center for Novel Silk Materials, Southwest University, \\ Chongqing 400715, China \\ * Correspondence: zslu@swu.edu.cn; Tel.: +86-23-6825-4732; Fax: +86-23-6825-4969
}

Received: 2 May 2018; Accepted: 21 May 2018; Published: 23 May 2018

\begin{abstract}
Electrospun nanofiber mats have been used as sensing elements to construct piezoresistive devices due to their large surface area and high porosity. However, they have not been utilized as skin-contact supporting layers to package conductive nanofiber networks for the fabrication of piezoresistive sensors. In this work, we developed a sandwich-structured pressure sensor, which can sensitively monitor human motions and vital signs, with electrospun nanofiber mats as supporting, sensing, and packaging layers. The nanofiber mats were prepared by electrospinning with biocompatible poly (L-lactide) (PLA), silk fibroin (SF), and collagen (COL) as raw materials. The synthesized PLA-SF-COL mat possesses a non-woven structure with a fiber diameter of $122 \pm 28 \mathrm{~nm}$ and a film thickness of $37 \pm 5.3 \mu \mathrm{m}$. Polypyrole (PPy) nanoparticles were grown in-situ on the mat to form a conductive layer. After stacking the pristine and conductive mats to form a PLA-SF-COL mat/(PPy-coated mat $)_{2}$ structure, another layer was electrospun to pack the multilayers for the construction of a sandwich-structured piezoresistive sensor. The as-prepared device can sensitively detect external pressures caused by coin loading and finger tapping/pressing. It can also tolerate more than 600 times of pressing without affecting its sensing capability. The human body-attached experiments further demonstrate that the sensor could real-time monitor finger/arm bending, arterial pulse, respiration rate, and speaking-caused throat vibration. The electrospinning-based fabrication may be used as a facile and low-cost strategy to produce flexible piezoresistive sensors with excellent skin-compatibility and great pressure sensing capability.
\end{abstract}

Keywords: piezoresistive sensor; electrospinning; wearable electronics; human motion; vital sign

\section{Introduction}

A wearable electronic device has been regarded as the best choice for everyday health monitoring due to its superior capability to real-time detect vital signs such as pulse rate, respiration rate, body temperature, and blood pressure [1-9]. The device can efficiently convert human physiological parameters into electronic signals, which are detectable, recordable, and analyzable with miniaturized instruments. Generally, wearable electronic pressure sensors can be divided into three types 
according to their working mechanisms: capacitive, piezoelectric, and piezoresistive sensors [10-14]. A microfabrication technique is needed to precisely control the opposite area and distance between the two electrodes in capacitive pressure sensors [10]. Although these sensors may provide relative high sensitivity, the complicated fabrication process greatly hinders their large-scale production and practical applications. Piezoelectric materials such as $\mathrm{ZnO}$ nanorod arrays and polyvinylidene fluoride (PVDF) films are used to functionalize flexible substrates for the preparation of piezoelectric sensors [5,11-15], which can generate electric charges in response to the applied mechanical stress. However, the construction of highly oriented 1-dimensional nanostructures and the adjustment of the polymer crystalline phase are very critical for the achievement of good piezoelectric effects.

In the past few years, piezoresistive pressure sensors based on flexible supports have been investigated as attractive wearable devices for human vital signs monitoring [16-20]. A typical one is fabricated by stacking flexible substrates and conductive sensing layers to form a sandwiched structure. Various materials including metal nanoparticles [16], carbonized fabrics [17], carbon nanofibers [18], and polymers [19] have been utilized to prepare the conductive sensing layers in the sandwich-structured sensors. Under mechanical stress, the conductive layers are compressed to create more crosslinking points for fast electron transfer, resulting in device resistance reduction. Since microand nano-scale fibers could generate a large number of potential networking sites, conductive fiber networks were produced to enhance the sensing performance $[14,21,22]$.

The electrospinning technique is a facile and low-cost method to prepare nanoscale non-woven fiber scaffolds with high surface area and large porosity [23-33]. It has also been applied to construct piezoresistive sensors containing fiber network layers. Electrospun nanofibers have been assembled to produce 3-dimensional and ultralight sponges with tunable conductivity for a tactile pressure sensor [34]. Poly(3,4-ethylenedioxythiophene):poly(styrenesulfonate)-polyvinyl alcohol nanofibers have been electrospun on a Kapton substrate and encapsulated with polydimethylsiloxane (PDMS) to construct a piezoresistive strain sensor. The device could be stuck on a human finger for the detection of finger bending [21]. Electrospun poly(4-vinylpyridine) nanofiber mats have been coated with a polyethylene terephthalate (PET) film and further micropatterned by lithography for the development of piezoresistive strain sensors [35]. A highly sensitive piezoresistive sensor consisting of PET substrate, electrospun polyaniline nanofibers, and Au-coated PDMS micropillars has been fabricated to measure neck pulse and wrist pulse [36]. Very recently, a freestanding carbon nanofiber mat, which was synthesized by carbonizing electrospun polyacrylonitrile network, was embedded with two layers of polyurethane to preparea piezoresistive sensor for human motion monitoring [18]. Unfortunately, besides electrospun nanofiber mats, flexible polymer substrates and packaging layers have been employed in all those reported devices.

To effectively detect human vital signs, the piezoresistive sensor has to be attached on human skin to sense the extremely weak vibration caused by blood flow and respiration. Therefore, in addition to the pressure sensing performance, skin affinity should be considered in device design. Polymers such as PET and PDMS could provide excellent flexibility and processability, but their biocompatibility and air/moisture permeability are still questionable. Electrospun nanofiber mats consisting of biocompatible polymers and natural proteins have already been utilized as degradable scaffolds for cell adhesion and proliferation in tissue engineering [37]. Moreover, their porous structures may enable the exchange of air/moisture between the skin and the environment. Electrospun nanofiber mats should be ideal supporting layers for skin-attached applications. However, to the best of our knowledge, electrospun nanofiber mats have not been utilized as skin-contacting layers to sandwich conductive nanofiber networks for a piezoresistive sensor.

In this work, we fabricated a flexible, sensitive, and skin-compatible sandwich-structured piezoresistive pressure sensor with electrospun fiber mats as supporting, sensing, and encapsulating layers. The substrate and packaging layers were electrospun nanofiber films consisting of eco-friendly poly(L-lactide) (PLA), collagen (COL), and silk fibroin (SF). The active layers are PLA-SF-COL films coated with polypyrrole (PPy). The surface morphologies and components of the films were 
characterized with scanning electron microscopy (SEM), X-ray diffraction (XRD), and attenuated total reflection-Fourier transfer infrared spectroscopy (ATR-FTIR), respectively. The pressure sensing property and durability were tested to estimate the performance of the as-prepared devices. The real-time measurements of human motions (finger/arm bending), vital signs (wrist/neck pulse and respiration rate), and throat vibration caused by speaking were conducted to demonstrate the great potential of the electrospun nanofiber mat-based pressure sensors in practical applications.

\section{Materials and Methods}

\subsection{Chemicals}

PLA pellets $(M w=150 \mathrm{kDa}, 99 \%), \mathrm{SF}(M w=50 \mathrm{kDa}, 99.99 \%)$ and COL $(M w=120 \mathrm{kDa}$, 99\%) were kindly provided by the Department of Orthopedics, Chongqing Hospital of Traditional Chinese Medicine. Pyrrole (Py, 99\%) was purchased from Adamas-beta (Shanghai, China). Iron chloride hexahydrate $\left(\mathrm{FeCl}_{3} \cdot 6 \mathrm{H}_{2} \mathrm{O}, 99 \%\right), p$-toluenesulfonic acid sodium salt $(96 \%)$ and 1,1,1,3,3,3-hexafluoro-2-propanol (HFIP, 99.5\%) were bought from Aladdin Reagent Co. (Shanghai, China). All other chemicals were of analytical grade and directly used without further purification in this study. Deionized water was produced by a Millipore water purification system.

\subsection{Preparation of PLA-SF-COL Nanofiber Mats by Electrospinning}

The PLA-SF-COL nanofiber mats were prepared by the Wang's route [37]. PLA pellets, SF, and COL were successively added into HFIP at weight percentages of $4 \%, 2 \%$, and $2 \%$, respectively [37]. The mixture was stirred at room temperature for $6 \mathrm{~h}$ to thoroughly dissolve the above solutes. A microinjection pump, a high-voltage power supply, and a grounded drum were used to construct a homemade electrospinning system. The as-prepared precursor solution was fed into a blunt needle with an inner diameter of $0.6 \mathrm{~mm}$ at a constant speed of $0.5 \mathrm{~mL} / \mathrm{h}$ under a $20 \mathrm{kV}$ voltage. The grounded drum with a diameter of $8 \mathrm{~cm}$, which rotates at a speed of $1000 \mathrm{rpm} / \mathrm{min}$, was placed $20 \mathrm{~cm}$ far from the blunt needle tip to collect the nanofibers (Scheme 1).

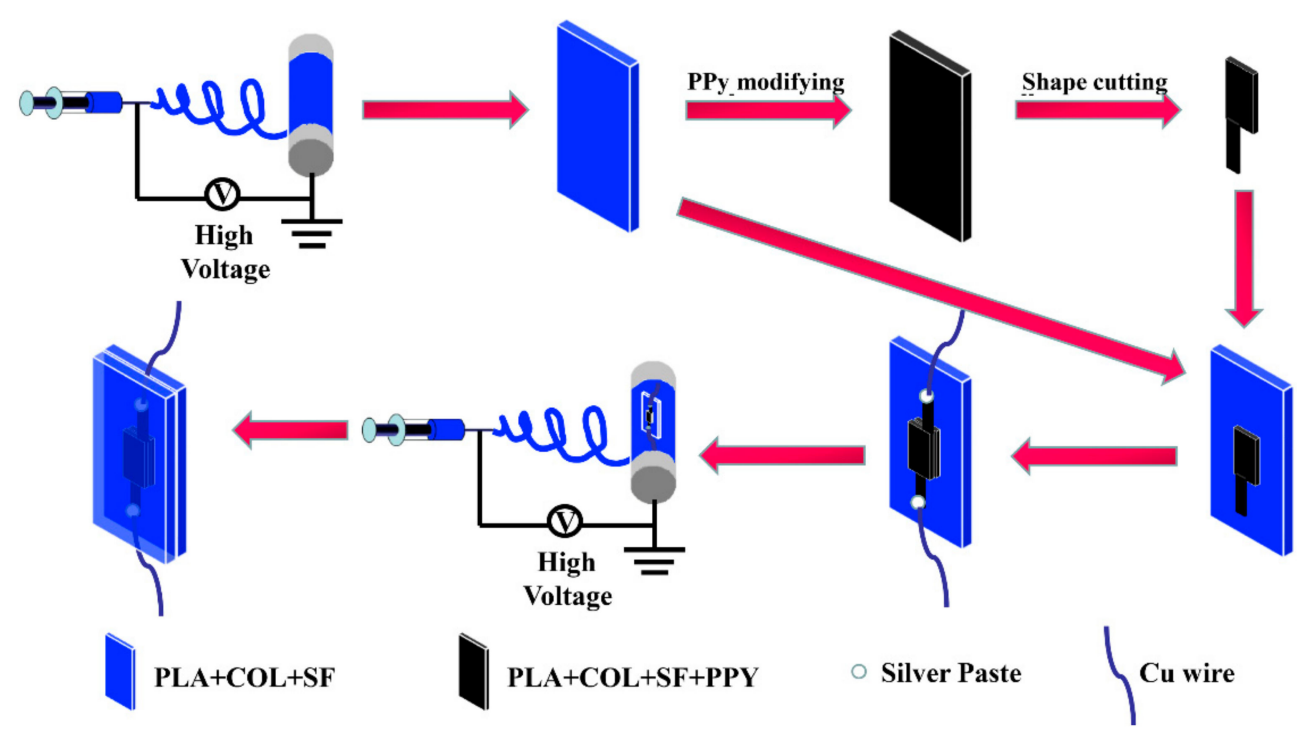

Scheme 1. Fabrication of a piezoresistive sensor based on electrospun nanofiber mats.

\subsection{Fabrication of the Conductive Layers}

The freshly prepared PLA-SF-COL nanofiber mats were removed from the drum and soaked in an aqueous solution containing $p$-toluenesulfonic acid sodium salt (dopant) and iron chloride hexahydrate (catalyst) at room temperature for $2 \mathrm{~h}$. Then, Py monomer was added into the above 
solution, holding at room temperature for different durations (2 to $10 \mathrm{~h}$ ) [38]. To optimize the Py amount for the achievement of highly conductive films, the Py concentration in the reaction system was changed from 7.5 to $30 \mathrm{mmol} / \mathrm{L}$, while maintaining the molar proportion of Py, catalyst and dopant as 1:0.91:0.39. The treated mats were washed with deionized water three times to thoroughly remove superfluous polymers. After drying at $40{ }^{\circ} \mathrm{C}$, they were cut into flag-shaped pieces with active area of $1 \mathrm{~cm} \times 1 \mathrm{~cm}$ (Scheme 1 ).

\subsection{Construction of Sandwich-Structured Piezoresistive Sensors}

The designed piezoresistive sensor consists of two supporting films and two conductive layers, all of which are electrospun nanofiber mats. The as-prepared PLA-SF-COL electrospun mat was knifed into a $3 \mathrm{~cm} \times 4 \mathrm{~cm}$ rectangle film and fixed on the grounded drum to serve as the first supporting layer. Two pieces of flag-shaped PPy-coated mats were stacked face-to-face to form a sensing element with a size of $1 \mathrm{~cm} \times 1 \mathrm{~cm}$. Copper wires were adhered to the ends of the flags with silver paste for later connecting with the measurement instruments. The sensing element was attached in the center of the first supporting layer. Another layer of PLA-SF-COL mat was spun on top of the sensing elements serving as both second supporting layer and sealing material. After clipping, a final piezoresistive sensor with a size of $3 \mathrm{~cm} \times 4 \mathrm{~cm}$ was produced (Scheme 1).

\subsection{Characterizations and Measurements}

Morphologies of PLA-SF-COL nanofiber mats before/after each modification were characterized using a JSM-7600 FESEM (JEOL, Tokyo, Japan). The ATR-FTIR (Nicolet 6700 FTIR, Thermo Electronic Corporation, Waltham, MA, USA) and XRD spectra (XRD-7000, Shimadzu, Japan) were examined to confirm the formation of the nanofiber mats. The sensing performances of the device were collected using an electrochemical workstation (CHI 660E, CH Instrument Company, Shanghai, China). During the measurements, the devices were fixed on the apparatus, which consists of a positioning system and a force sensor (Figure S1). Pressure-induced resistance changes were collected under an operating voltage of $1.0 \mathrm{~V}$ to evaluate the sensing performance. Body motions, vital signs, and voices of a female volunteer were real-time measured to demonstrate the feasibility of the sensor. Since the operation voltage was quite low, the current response of the device was set at a level of dozens of microamperes, which would not cause adverse effects to the volunteer.

\section{Results and Discussion}

PLA, SF, and COL were mixed together to prepare a precursor solution for the electrospinning of biocompatible nanofiber mats. As shown in Figure 1A,B, fibers with an average diameter of $122 \pm 28 \mathrm{~nm}$ crosslink with each other to form a non-woven structure, which is very typical morphology of an electrospun mat. The thickness of the collected mat was about $37.0 \pm 5.3 \mu \mathrm{m}$ (Figure S2), indicating that the mat could be utilized as a freestanding film for the following PPy modification and sensor assembly. To prepare the sensing layer, the as-prepared mats were immersed in a solution containing catalysts, dopants, and Py monomers for the in-situ growth of PPy. In order to achieve high conductivity, the concentration of Py monomers and the polymerization time were optimized, respectively. It was found that the mats with the highest conductivity could be obtained in the system containing $15 \mathrm{mM}$ Py monomer after $6 \mathrm{~h}$ of reaction (Figure S3). The modified mat was covered by agglomerated nanoparticles and the crosslinking fibers could only be seen from the gaps (Figure 1C). Figure 1D,E illustrate the morphologies of PPy-modified nanofibers and PPy stacking on the mats, respectively. In comparison to the relatively smooth surface of PLA-SF-COL fibers, a number of nanoparticles were uniformly coated on the modified fibers (Figure 1D), causing significant increment of the fiber diameter. The amorphous stacking structure could also be observed on the mat surface, which matches well with the morphology of PPy film (Figure 1E) [39]. The cross-sectional SEM image of a modified sample (Figure S4) further confirms the deposition of PPy throughout the whole mat. During the polymerization, PPy may first grow on the fiber surface to form a conductive 
layer, followed by stacking on the mat to produce amorphous agglomerates (Figure S5). The PPy layers on the fibers and surface-attached PPy agglomerates may both contribute to the conductivity of the PLA-SF-COL-based sensing elements.
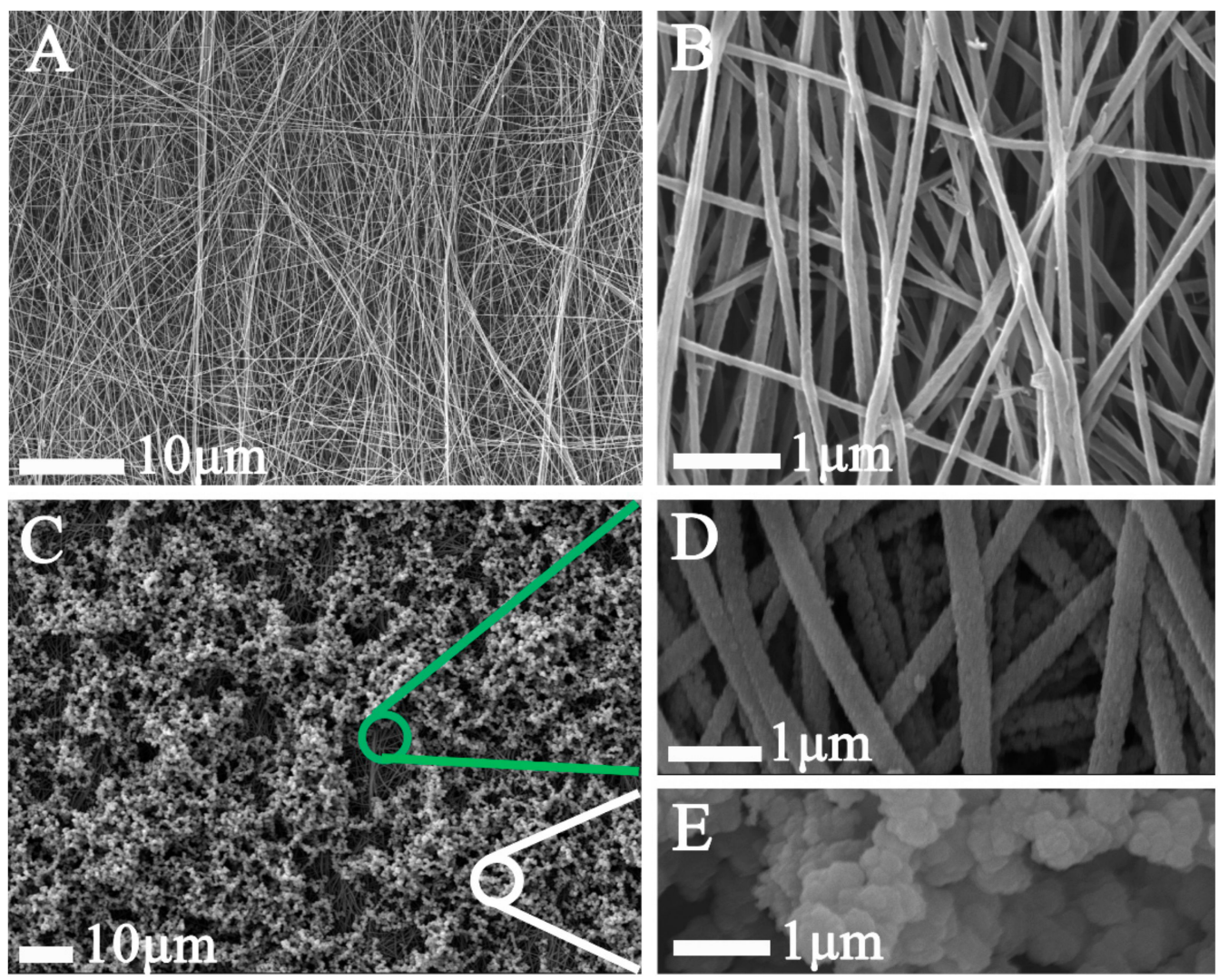

Figure 1. Scanning electron microscopy (SEM) images of an electrospun PLA-SF-COL nanofiber mat at low (A) and high magnifications (B); (C) A PLA-SF-COL nanofiber mat after PPy modification; (D-E) SEM images of PPy-immobilized PLA-SF-COL nanofiber mat at high magnifications.

XRD and ATR-FTIR were carried out to verify components of the electrospun nanofiber mats and the successful immobilization of PPy. The XRD spectrum of PLA-SF-COL mats (Figure 2A) displays two prominent diffraction peaks at $16.4^{\circ}$ and $23^{\circ}$, which can be assigned to the reflection of crystalline PLA and SF, respectively [40-42]. No clear peak for COL can be identified from the curve. Normally, COL possesses a wide and weak peak at around $20^{\circ}$ [43], which may be masked by the strong diffraction patterns of PLA and SF. As to the PPy-modified mat, a broad peak in the range of $20-30^{\circ}$ can be observed, suggesting the coverage of the amorphous polymers on the mat surface $[44,45]$. Interestingly, the peaks for PLA, SF, and COL could not be found in the XRD pattern of the PPy-modified mats. The phenomenon may be caused by the full coverage of a thick PPy layer on the mats [46-48]. Figure 2B shows the FTIR spectra of the electrospun mats before and after PPy functionalization, respectively. The weak bands at 1751 and $1087 \mathrm{~cm}^{-1}$ correspond to the backbone ester groups of the PLA [49,50]. The absorptions at 1622 and $1264 \mathrm{~cm}^{-1}$ indicate the existence of $\mathrm{SF}$ in the mat, while COL contributes to the band at $1454 \mathrm{~cm}^{-1}$ [51,52]. After PPy immobilization, some new absorption bands appear in the spectrum. The band at $1543 \mathrm{~cm}^{-1}$ could be attributed to pyrrole rings. The peaks at 1300 and $1041 \mathrm{~cm}^{-1}$ come from the deforming vibration of $\mathrm{N}-\mathrm{H}$ and $\mathrm{C}-\mathrm{H}$ as well as stretching vibration of $\mathrm{C}-\mathrm{C}$, respectively [53]. The results undoubtedly prove that the electrospun nanofiber mats are composed of PLA, SF, and COL. After in-situ polymerization, PPy could be deposited on the mats to form fiber-adsorbed and surface-attached structures. 

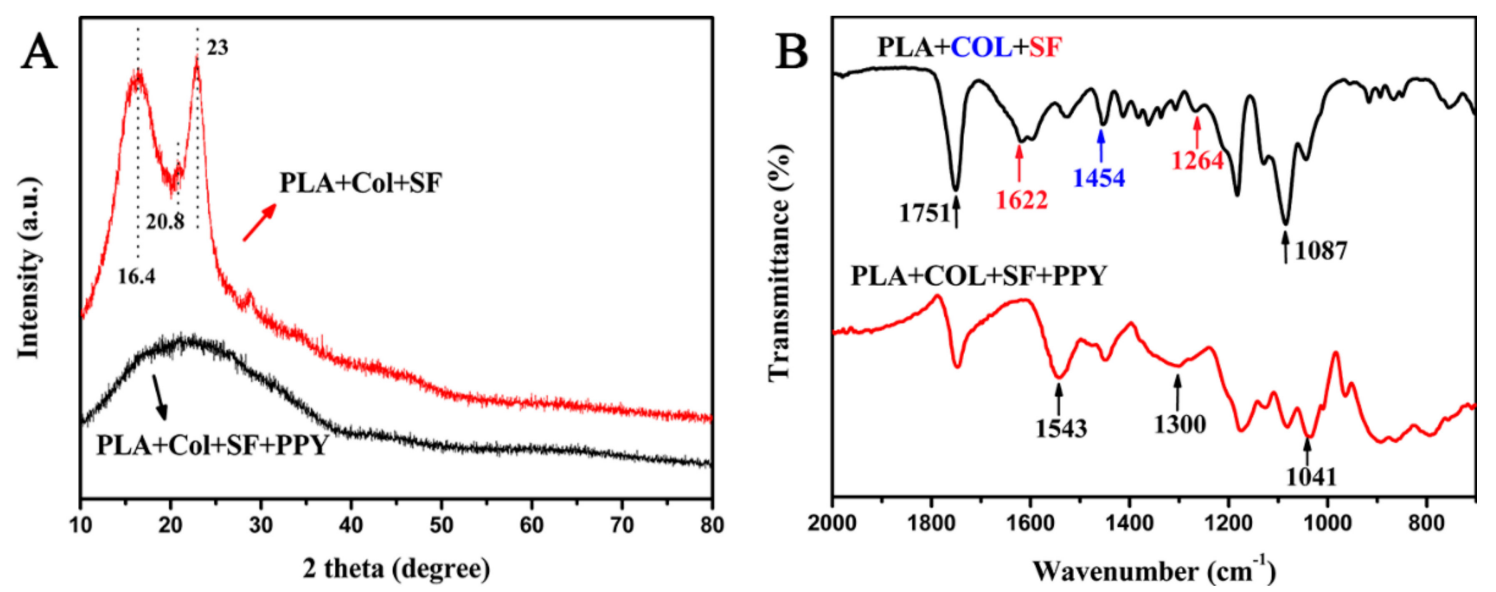

Figure 2. X-ray diffraction (XRD) patterns (A) and Fourier transfer infrared spectroscopy (FTIR) spectra (B) of PLA-SF-COL nanofiber mats before and after PPy modification, respectively.

The as-prepared non-modified and PPy-coated PLA-SF-COL mats were employed to construct sandwich-structured piezoresistive sensors (Figure 3A). The color of the electrospun mats changes from white to black during the PPy immobilization process. After cutting into the desired size and shape, one PPy-functionalized film was attached on a PLA-SF-COL supporting layer. Then, another piece of conductive film was stacked on the top of it to form a sensing element. Two copper wires were adhered at the ends of the top and bottom conductive films, respectively, for later connecting to the testing instruments. The system was fixed on a grounded drum for another round of electrospinning. The deposited nanofiber mat serves as both packaging material and top supporting layer (Figure 3B,C). Finally, a sandwich-structured piezoresistive sensor with nanofiber mats as supporting, sensing, and packaging layers could be obtained for the pressure-response tests and human vital sign detection. The sensing mechanism for the nanofiber mat-based piezoresistive devices could be explained by the change of the number of contacting points during the loading- unloading process (Figure S6). At the unloading state, there are less contact points between the top and bottom conductive layers, thus causing a relatively larger device resistance. On the contrary, external pressure may push the two conductive layers much tighter together to produce more contacting sites, resulting in the reduction of device resistance.

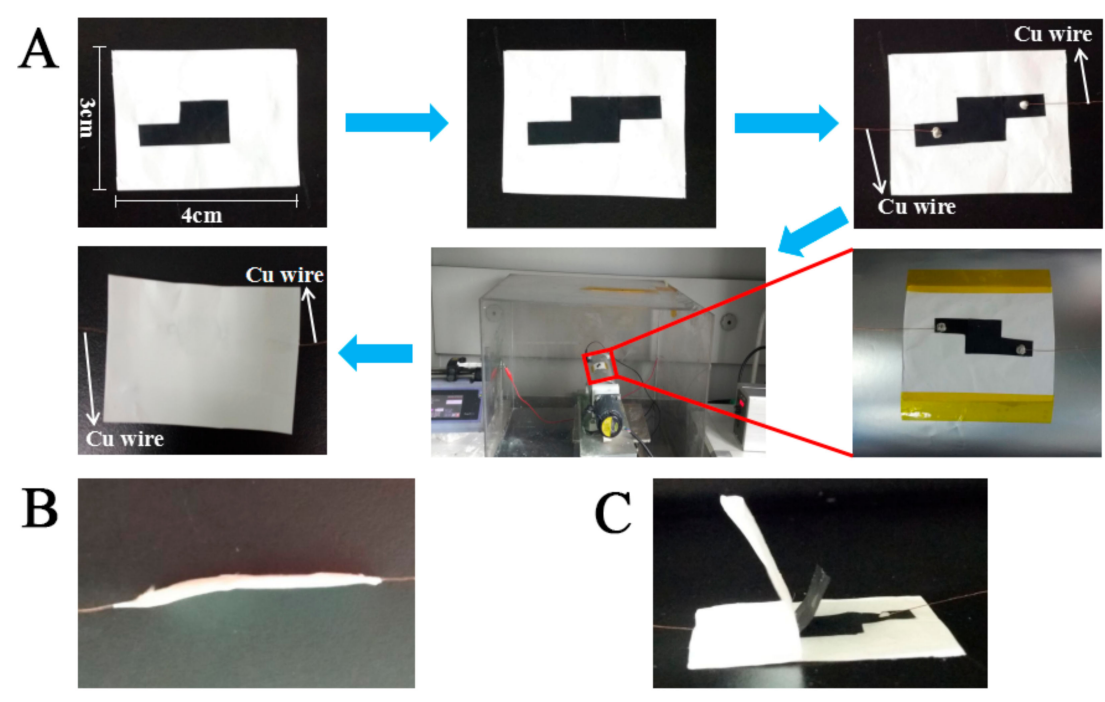

Figure 3. (A) Fabrication of a sandwich structured piezoresistive sensor with nanofiber mats as supporting, sensing, and packaging layers via electrospinning; (B) Side view of an as-prepared device; (C) Side view of a disassembled device. 
The pressure-dependent resistance change is exhibited in Figure 4A. In the low-pressure region (from 0 to $1.27 \mathrm{~Pa}$ ), the resistance change increases linearly with the applied pressure. The sensitivity in this region is as high as $24.13 \mathrm{kPa}^{-1}$ with high linearity of $R^{2}=0.99$ (Inset of Figure 4A). Based on the experimental data and statistical analysis, the limit of detection was determined to be $1.00 \mathrm{~Pa}$. The current-voltage curves of the device under different pressures show Ohmic behavior (Figure S7). To further investigate the influence of humidity on the device performance, the resistance changes caused by certain pressures under different humidities were tested. As the relative humidity increases from $47 \%$ to $56 \%$, the resistance change gradually reduces (Figure S8), suggesting that the quantitative measurement of external pressures should be conducted under an environment with constant humidity. In practical application, the humidity change seems to be inevitable. A re-calibration process may be needed to ensure the accuracy of the quantitative analysis. Human finger tapping and pressing were real-time monitored using the electrospun mats-based sensors with a force at about $3 \mathrm{~N}$ (Figure 4B,C). The resistive responses match well with the repetitive tapping and pressing. The external pressure induces a very rapid drop in the device resistance. When the pressure is maintained on the device surface, the resistance stays at a constant level. Once the force is removed, the resistance recovers to the original level. As the tapping frequency increases from 0.1 to $3.5 \mathrm{~Hz}$, the device could provide satisfactory electrical responses (Figure S9). To further demonstrate the sensitivity of the sensor, four coins were stacked on the top of a device and then removed one by one (Figure 4D). The loading of four coins significantly decreased the device relative resistance to $\sim 0.82$. During the removal process, the relative resistance gradually increased and finally went back to $\sim 1.0$, indicating that the sensor could precisely detect the external pressure change. It was of interest to find that the resistance alterations caused by the 4th and 3rd coins were obviously smaller than those caused by the 2nd and 1st ones. The phenomenon may suggest that the sensor is more sensitive to a relative small pressure. The micro- and nano-scale features of the PPy conductive layers may contribute to the low detection limit, high sensitivity, and fast response of the as-prepared devices. Since durability and stability are critical parameters for a flexible device, responses of the device to repetitive pressing were real-time tested in the present study (Figure 4E,F). The signals perfectly matched with the pressing during the measurement. Moreover, both relative resistances at loading and unloading states kept quite stable at $\sim 0.89$ and $\sim 1.0$, respectively. The results show that the electrospun mat-based sensors may tolerate more than 600 times of pressing without affecting their pressure sensing capability. To rule out the interference of the piezoelectric effects from PLA, a device without PPy layers was tested. As exhibited in Figure S10, the current signals are negligible in comparison with the ones obtained from a PPy-incorporated device. The sensing performances of three devices were also examined to show the run-to-run difference of the fabrication process (Figure S11). After 7 and 14 days of storage at ambient conditions, there was no significant change on the electric response to a certain pressure, demonstrating the great long-term stability of the as-prepared devices (Figure S12). In our study, we found that both size and shape of the active component could significantly affect the performance of the sensing devices. Further studies may be conducted to systematically investigate the size and shape effects of the active components on the performance of piezoresistive sensors. 

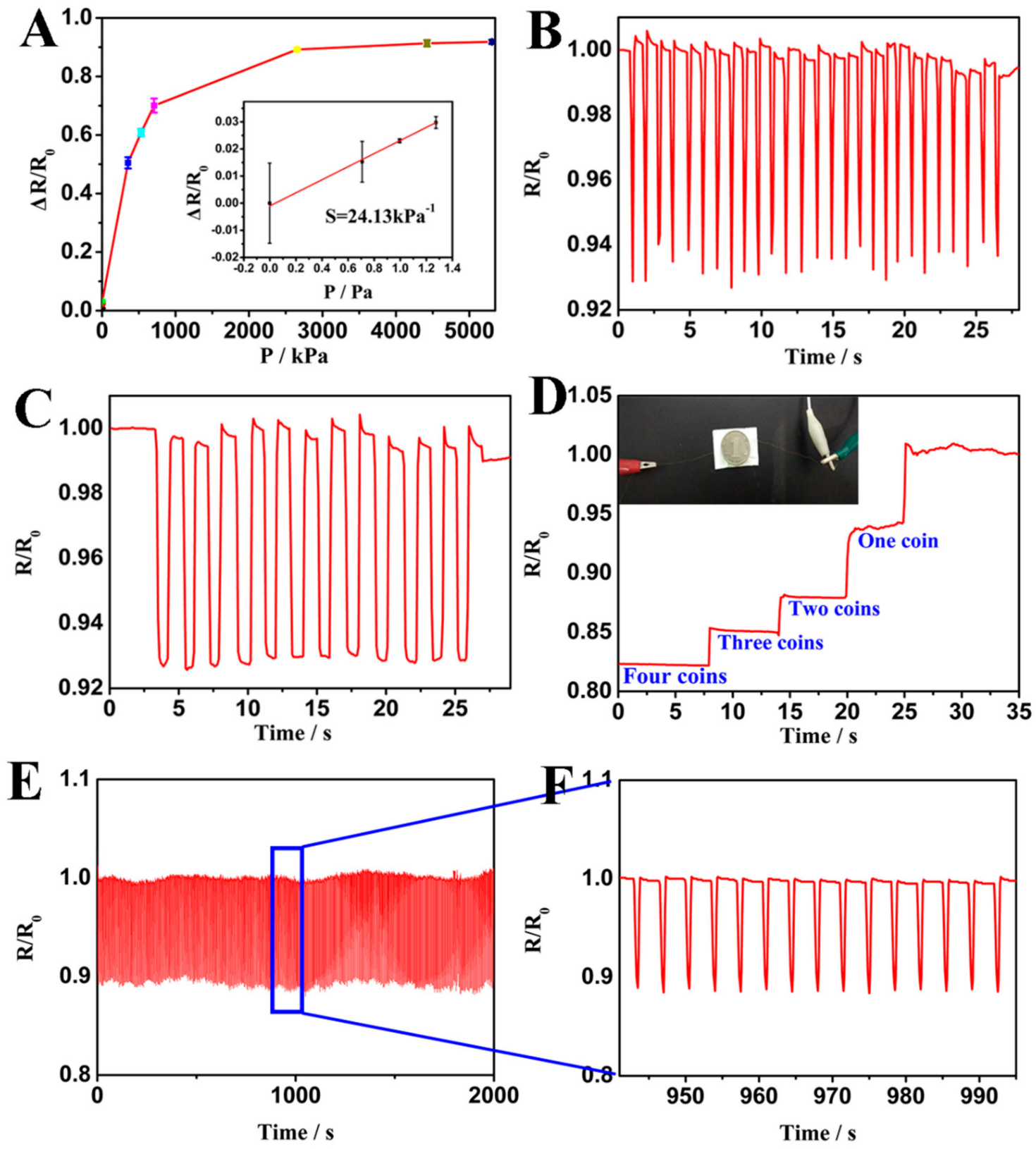

Figure 4. (A) Resistance change-pressure relationship; Responses of the sensor to finger tapping (B) and pressing (C); (D) Relative resistance of the sensor after loading different numbers of coins; (E) Responses of the nanofiber mats-based piezoresistive sensor upon repetitive loading and unloading of a constant pressure; (F) An enlarged view of the selected part.

The electrospun mat-based piezoresistive devices were fastened on the joints of thumb and arm for the measurement of human motions (Figure 5). When the thumb and arm are kept straight, there is no pressure on the attached device and the relative resistance has no alteration. Once the volunteer bends the thumb and arm, extrusion force is applied to the sensor, leading to the sharp resistance decreases observed in real-time curves. As the thumb and arm are straightened again, the device resistances return to the original level. It was noted that the signals may vary in intensity and pattern during the repetitive bending tests, which may have been caused by unstable contact between the two conductive layers. 

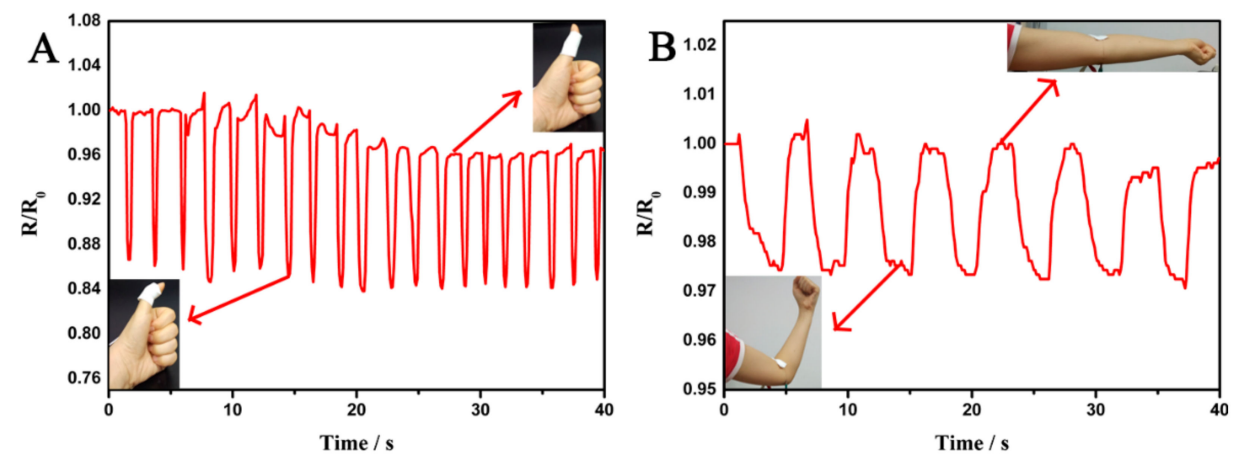

Figure 5. Responses of the nanofiber mats-based piezoresistive sensor to human thumb bending (A) and arm bending (B).

Cardiac contraction and relaxation will respectively cause distension and compression of an artery, which may be sensed by the skin-attached sensors. In the present study, the as-prepared devices were mounted on a human wrist and neck for real-time monitoring wrist (Video S1) and neck pulses (Figure $6 \mathrm{~A}, \mathrm{~B}$ ). The initial resistance of the device was $283.1 \pm 0.6 \mathrm{k} \Omega$. The sensor could easily detect signals corresponding to the wrist and neck pulses. Moreover, the wrist and neck pulses could be calculated based on the real-time curves as 66 times/min and 72 times/min, respectively, which fall in the range of a healthy female (60-100 times/min). A control experiment was also conducted in our study for easy comparison (Figure S13). The device was also attached on the volunteer's abdomen to sense the up and down movement caused by breathing. The resistance reduction and recovery in Figure $6 \mathrm{C}$ stand for the inhalation and exhalation of the volunteer, respectively. Within $20 \mathrm{~s}, 10$ occasions of breathing could be detected. The results demonstrate that the electrospun mat-based piezoresistive sensors should be very promising in real-time monitoring of human vital signs such as pulse and respiration.
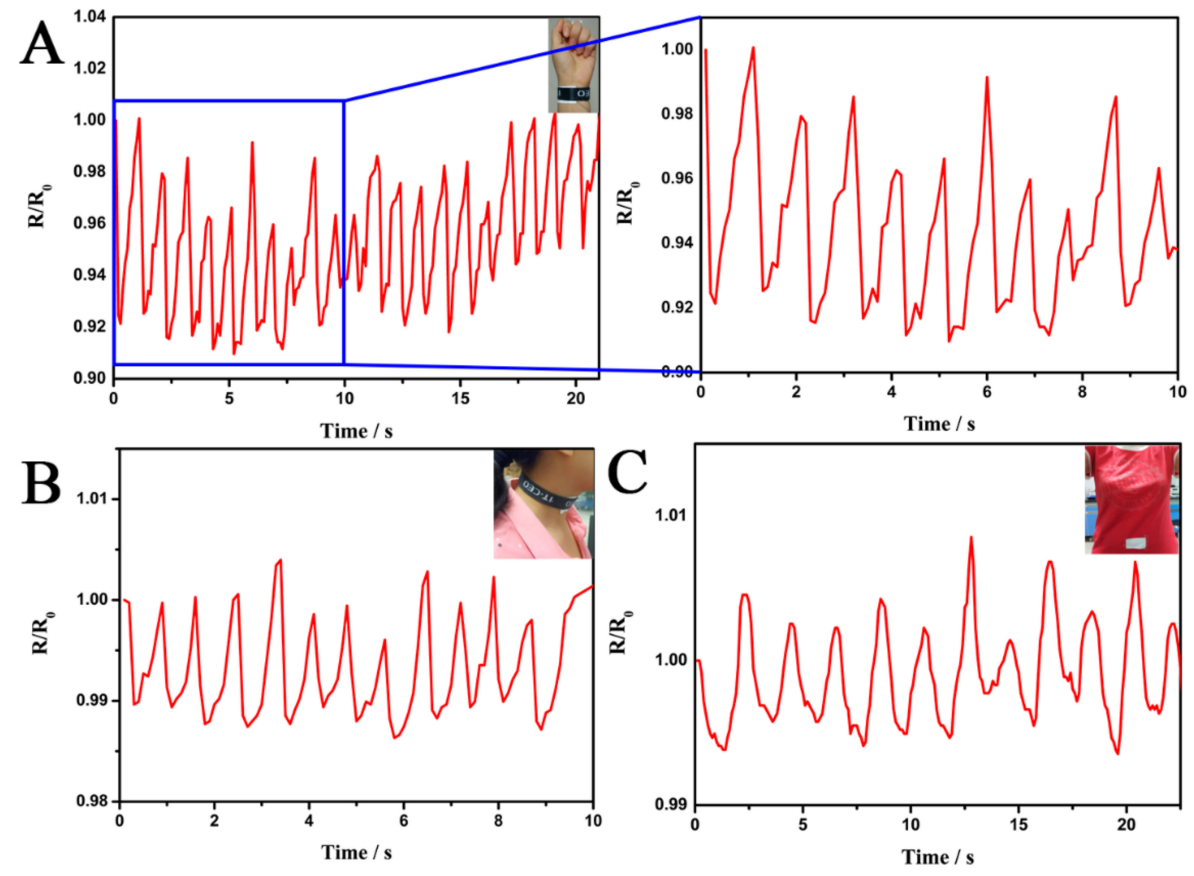

Figure 6. Real-time measurements of human wrist pulse (A), neck pulse (B) and respiration rate (C) using the nanofiber mats-based piezoresistive sensor. Insets: Attachments of the sensor on the volunteer's wrist, neck, and abdomen, respectively. 
We also tested the responses of the device to the sound change by attaching it on the human throat. When the subject was speaking the word "Hello", one signal could be immediately discovered in the real-time curve (Figure 7A). However, when the subject was speaking two words "Southwest University", two well-defined waves, corresponding to the words, appeared (Figure 7B). It is obvious that the sensor could catch the vibration of the throat during the speaking process. Therefore, the sensor may be potentially applied to differentiate human voices and to convert acoustic information into electronic signals in the future.
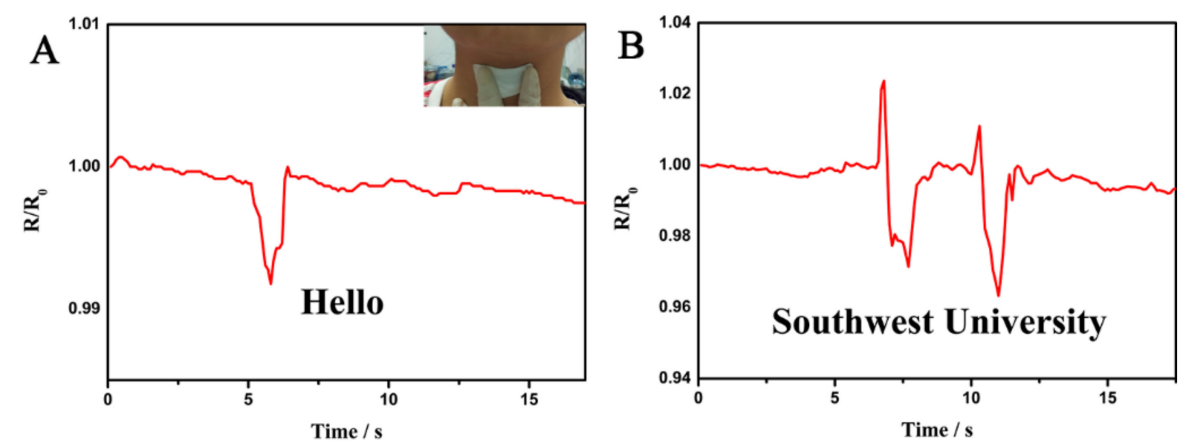

Figure 7. Real-time measurements of vocal-cord vibrations using a throat-attached nanofiber mat-based sensor when the volunteer is speaking "Hello" (A) and "Southwest University" (B), respectively.

\section{Conclusions}

In summary, we successfully fabricated a sandwich-structured piezoresistive sensor with electrospun nanofiber mats as supporting, sensing, and packaging layers. Firstly, biocompatible PLA-SF-COL nanofiber mats were prepared via electrospinning to serve as the bottom supporting layers and the matrices for PPy modification. Then, the pre-cut PLA-SF-COL mats were immersed in Py monomer solution for the in-situ growth of conductive polymers. The non-woven structure and the biocompatible components of the mats as well as PPy immobilization were verified with SEM, XRD, and FTIR, respectively. After stacking the materials on the drum to form a PLA-SF-COL mat/(PPy-coated mat $)_{2}$ structure, another PLA-SF-COL mat was deposited on its surface to function as both top supporting layer and packaging layer. The as-prepared device is a freestanding multilayered film with great flexibility and good biocompatibility. After a series of tests, the piezoresistive sensor showed high sensitivity to external pressures and excellent durability to repetitive pressing. With these advantages, it could be applied to monitor finger/arm bending, arterial pulse, respiration rate, as well as speaking-caused throat vibration. This work may not only provide a facile electrospinning-based approach to constructing flexible piezoresistive sensors, but also for use to develop a skin-compatible wearable sensor with great potential in practical real-time monitoring of human motions and vital signs.

Supplementary Materials: The supporting data are available online at http:/ /www.mdpi.com/2073-4360/10/6/575/ s1.

Author Contributions: Conceptualization, Y.Q. and Q.X.; data curation, Z.Z., B.L. and L.X.; formal analysis, Z.Z., B.L., L.X. and F.W.; funding acquisition, Z.L.; investigation, B.L. and Z.L.; supervision, Z.L.; writing-original draft, Z.Z., L.X. and Z.L.; writing-review and editing, Y.Q., F.W., Q.X., and Z.L.

Acknowledgments: This work is financially supported by Chongqing Engineering Research Center for Micro-Nano Biomedical Materials and Devices.

Conflicts of Interest: The authors declare no conflict of interest.

\section{References}

1. Dagdeviren, C.; Shi, Y.; Joe, P.; Ghaffari, R.; Balooch, G.; Usgaonkar, K.; Gur, O.; Tran, P.L.; Crosby, J.R.; Meyer, M.; et al. Conformal piezoelectric systems for clinical and experimental characterization of soft tissue biomechanics. Nat. Mater. 2015, 14, 728-736. [CrossRef] [PubMed] 
2. Wang, Y.; Wang, L.; Yang, T.; Li, X.; Zang, X.; Zhu, M.; Wang, K.; Wu, D.; Zhu, H. Wearable and highly sensitive graphene strain sensors for human motion monitoring. Adv. Funct. Mater. 2014, 24, 4666-4670. [CrossRef]

3. Wang, X.; Gu, Y.; Xiong, Z.; Cui, Z.; Zhang, T. Silk-molded flexible, ultrasensitive, and highly stable electronic skin for monitoring human physiological signals. Adv. Mater. 2014, 26, 1336-1342. [CrossRef] [PubMed]

4. Roh, E.; Hwang, B.-U.; Kim, D.; Bo-Yeong, K.; Lee, N.-E. Stretchable, transparent, ultrasensitive, and patchable strain sensor for human-machine interfaces comprising a nanohybrid of carbon nanotubes and conductive elastomers. ACS Nano 2015, 9, 6252-6261. [CrossRef] [PubMed]

5. Chen, X.; Shao, J.; An, N.; Li, X.; Tian, H.; Xu, C.; Ding, Y. Self-powered flexible pressure sensors with vertically well-aligned piezoelectric nanowire arrays for monitoring vital signs. J. Mater. Chem. C 2015, 3, 11806-11814. [CrossRef]

6. Pang, C.; Lee, G.Y.; Kim, T.I.; Kim, S.M.; Kim, H.N.; Ahn, S.H.; Suh, K.Y. A flexible and highly sensitive strain-gauge sensor using reversible interlocking of nanofibres. Nat. Mater. 2012, 11, 795-801. [CrossRef] [PubMed]

7. Dagdeviren, C.; Su, Y.; Joe, P.; Yona, R.; Liu, Y.; Kim, Y.S.; Huang, Y.; Damadoran, A.R.; Xia, J.; Martin, L.W.; et al. Conformable amplified lead zirconate titanate sensors with enhanced piezoelectric response for cutaneous pressure monitoring. Nat. Commun. 2014, 5, 4496. [CrossRef] [PubMed]

8. Yang, J.; Wei, D.; Tang, L.; Song, X.; Luo, W.; Chu, J.; Gao, T.; Shi, H.; Du, C. Wearable temperature sensor based on graphene nanowalls. RSC Adv. 2015, 5, 25609-25615. [CrossRef]

9. Lu, Z.; Zhang, H.; Mao, C.; Li, C.M. Silk fabric-based wearable thermoelectric generator for energy harvesting from the human body. Appl. Energy 2016, 164, 57-63. [CrossRef]

10. Zhao, X.; Hua, Q.; Yu, R.; Zhang, Y.; Pan, C. Flexible, stretchable and wearable multifunctional sensor array as artificial electronic skin for static and dynamic strain mapping. Adv. Electron. Mater. 2015, 1, 1500142. [CrossRef]

11. Seung, W.; Gupta, M.K.; Lee, K.Y.; Shin, K.-S.; Lee, J.-H.; Kim, T.Y.; Kim, S.; Lin, J.; Kim, J.H.; Kim, S.-W. Nanopatterned textile-based wearable triboelectric nanogenerator. ACS Nano 2015, 9, 3501-3509. [CrossRef] [PubMed]

12. Lee, M.; Chen, C.Y.; Wang, S.; Cha, S.N.; Park, Y.J.; Kim, J.M.; Chou, L.J.; Wang, Z.L. A hybrid piezoelectric structure for wearable nanogenerators. Adv. Mater. 2012, 24, 1759-1764. [CrossRef] [PubMed]

13. Qiu, Y.; Lei, J.; Yang, D.; Yin, B.; Zhang, H.; Bian, J.; Ji, J.; Liu, Y.; Zhao, Y.; Luo, Y.; et al. Enhanced performance of wearable piezoelectric nanogenerator fabricated by two-step hydrothermal process. Appl. Phys. Lett. 2014, 104, 113903. [CrossRef]

14. Lou, Z.; Chen, S.; Wang, L.; Jiang, K.; Shen, G. An ultra-sensitive and rapid response speed graphene pressure sensors for electronic skin and health monitoring. Nano Energy 2016, 23, 7-14. [CrossRef]

15. Mao, C.; Zhang, H.; Lu, Z. Flexible and wearable electronic silk fabrics for human physiological monitoring. Smart Mater. Struct. 2017, 26, 095033. [CrossRef]

16. Tee, B.C.; Wang, C.; Allen, R.; Bao, Z. An electrically and mechanically self-healing composite with pressureand flexion-sensitive properties for electronic skin applications. Nat. Nanotechnol. 2012, 7, 825-832. [CrossRef] [PubMed]

17. Wang, C.; Li, X.; Gao, E.; Jian, M.; Xia, K.; Wang, Q.; Xu, Z.; Ren, T.; Zhang, Y. Carbonized silk fabric for ultrastretchable, highly sensitive, and wearable strain sensors. Adv. Mater. 2016, 28, 6640-6648. [CrossRef] [PubMed]

18. Ding, Y.; Yang, J.; Tolle, C.R.; Zhu, Z. A highly stretchable strain sensor based on electrospun carbon nanofibers for human motion monitoring. RSC Adv. 2016, 6, 79114-79120. [CrossRef]

19. Oliva-Avilés, A.I.; Avilés, F.; Sosa, V. Electrical and piezoresistive properties of multi-walled carbon nanotube/polymer composite films aligned by an electric field. Carbon 2011, 49, 2989-2997. [CrossRef]

20. Ding, Y.; Yang, J.; Tolle, C.R.; Zhu, Z. Flexible and compressible PEDOT:PSS@melamine conductive sponge prepared via one-step dip coating as piezoresistive pressure sensor for human motion detection. ACS Appl. Mater. Interfaces 2018, 10, 16077-16086. [CrossRef] [PubMed]

21. Liu, N.; Fang, G.; Wan, J.; Zhou, H.; Long, H.; Zhao, X. Electrospun pedot:Pss-pva nanofiber based ultrahigh-strain sensors with controllable electrical conductivity. J. Mater. Chem. 2011, 21, 18962. [CrossRef]

22. Gao, Q.; Meguro, H.; Okamoto, S.; Kimura, M. Flexible tactile sensor using the reversible deformation of poly(3-hexylthiophene) nanofiber assemblies. Langmuir 2012, 28, 17593-17596. [CrossRef] [PubMed] 
23. Chronakis, I.S.; Grapenson, S.; Jakob, A. Conductive polypyrrole nanofibers via electrospinning: Electrical and morphological properties. Polymer 2006, 47, 1597-1603. [CrossRef]

24. Li, C.; Vepari, C.; Jin, H.J.; Kim, H.J.; Kaplan, D.L. Electrospun silk-BMP-2 scaffolds for bone tissue engineering. Biomaterials 2006, 27, 3115-3124. [CrossRef] [PubMed]

25. Min, B.-M.; Lee, G.; Kim, S.H.; Nam, Y.S.; Lee, T.S.; Park, W.H. Electrospinning of silk fibroin nanofibers and its effect on the adhesion and spreading of normal human keratinocytes and fibroblasts in vitro. Biomaterials 2004, 25, 1289-1297. [CrossRef] [PubMed]

26. Shao, S.; Zhou, S.; Li, L.; Li, J.; Luo, C.; Wang, J.; Li, X.; Weng, J. Osteoblast function on electrically conductive electrospun PLA/MWCNTs nanofibers. Biomaterials 2011, 32, 2821-2833. [CrossRef] [PubMed]

27. Jin, H. Human bone marrow stromal cell responses on electrospun silk fibroin mats. Biomaterials 2004, 25, 1039-1047. [CrossRef]

28. Jiang, S.; Chen, Y.; Duan, G.; Mei, C.; Greiner, A.; Agarwal, S. Electrospun nanofiber reinforced composites: A review. Polym. Chem. 2018, 9, 2685-2720. [CrossRef]

29. Greiner, A.; Wendorff, J.H. Electrospinning: A fascinating method for the preparation of ultrathin fibers. Angew. Chem. 2007, 46, 5670-5703. [CrossRef] [PubMed]

30. Jiang, S.; Helfricht, N.; Papastavrou, G.; Greiner, A.; Agarwal, S. Low-density self-assembled poly( $N$-isopropyl acrylamide) sponges with ultrahigh and extremely fast water uptake and release. Macromol. Rapid Commun. 2018, 39, e1700838. [CrossRef] [PubMed]

31. Cui, H.; Li, Y.; Zhao, X.; Yin, X.; Yu, J.; Ding, B. Multilevel porous structured polyvinylidene fluoride/polyurethane fibrous membranes for ultrahigh waterproof and breathable application. Compos. Commun. 2017, 6, 63-67. [CrossRef]

32. Jiang, S.; Duan, G.; Kuhn, U.; Morl, M.; Altstadt, V.; Yarin, A.L.; Greiner, A. Spongy gels by a top-down approach from polymer fibrous sponges. Angew. Chem. 2017, 56, 3285-3288. [CrossRef] [PubMed]

33. Zainab, G.; Iqbal, N.; Babar, A.A.; Huang, C.; Wang, X.; Yu, J.; Ding, B. Free-standing, spider-web-like polyamide/carbon nanotube composite nanofibrous membrane impregnated with polyethyleneimine for $\mathrm{CO}_{2}$ capture. Compos. Commun. 2017, 6, 41-47. [CrossRef]

34. Xu, T.; Ding, Y.; Wang, Z.; Zhao, Y.; Wu, W.; Fong, H.; Zhu, Z. Three-dimensional and ultralight sponges with tunable conductivity assembled from electrospun nanofibers for a highly sensitive tactile pressure sensor. J. Mater. Chem. C 2017, 5, 10288-10294. [CrossRef]

35. Saetia, K.; Schnorr, J.M.; Mannarino, M.M.; Kim, S.Y.; Rutledge, G.C.; Swager, T.M.; Hammond, P.T. Spray-layer-by-layer carbon nanotube/electrospun fiber electrodes for flexible chemiresistive sensor applications. Adv. Funct. Mater. 2014, 24, 492-502. [CrossRef]

36. Park, H.; Jeong, Y.R.; Yun, J.; Hong, S.Y.; Jin, S.; Lee, S.-J.; Zi, G.; Ha, J.S. Stretchable array of highly sensitive pressure sensors consisting of polyaniline nanofibers and Au-coated polydimethylsiloxane micropillars. ACS Nano 2015, 9, 9974-9985. [CrossRef] [PubMed]

37. Wang, G.; Hu, X.; Lin, W.; Dong, C.; Wu, H. Electrospun PLGA-silk fibroin-collagen nanofibrous scaffolds for nerve tissue engineering. In Vitro Cell. Dev. Biol. Anim. 2011, 47, 234-240. [CrossRef] [PubMed]

38. Cucchi, I.; Boschi, A.; Arosio, C.; Bertini, F.; Freddi, G.; Catellani, M. Bio-based conductive composites: Preparation and properties of polypyrrole (PPy)-coated silk fabrics. Synth. Met. 2009, 159, $246-253$. [CrossRef]

39. Li, K.; Zhang, H.; Tang, T.; Tang, Y.; Wang, Y.; Jia, J. Facile electrochemical polymerization of polypyrrole film applied as cathode material in dual rotating disk photo fuel cell. J. Power Sources 2016, 324, 368-377. [CrossRef]

40. Li, Y.; Chen, F.; Nie, J.; Yang, D. Electrospun poly(lactic acid)/chitosan core-shell structure nanofibers from homogeneous solution. Carbohydr. Polym. 2012, 90, 1445-1451. [CrossRef] [PubMed]

41. Abdelwahab, M.A.; Flynn, A.; Chiou, B.-S.; Imam, S.; Orts, W.; Chiellini, E. Thermal, mechanical and morphological characterization of plasticized PLA-PHB blends. Polym. Degrad. Stab. 2012, 97, 1822-1828. [CrossRef]

42. Kim, U.J.; Park, J.; Kim, H.J.; Wada, M.; Kaplan, D.L. Three-dimensional aqueous-derived biomaterial scaffolds from silk fibroin. Biomaterials 2005, 26, 2775-2785. [CrossRef] [PubMed]

43. Teng, S.-H.; Lee, E.-J.; Wang, P.; Kim, H.-E. Collagen/hydroxyapatite composite nanofibers by electrospinning. Mater. Lett. 2008, 62, 3055-3058. [CrossRef] 
44. Yuvaraj, H.; Park, E.J.; Gal, Y.-S.; Lim, K.T. Synthesis and characterization of polypyrrole-TiO 2 nanocomposites in supercritical $\mathrm{CO}_{2}$. Colloids Surf. A 2008, 313, 300-303. [CrossRef]

45. Chougule, M.A.; Pawar, S.G.; Godse, P.R.; Mulik, R.N.; Sen, S.; Patil, V.B. Synthesis and characterization of polypyrrole (PPy) thin films. Soft Nanosci. Lett. 2011, 1, 6-10. [CrossRef]

46. Babu, K.F.; Senthilkumar, R.; Noel, M.; Kulandainathan, M.A. Polypyrrole microstructure deposited by chemical and electrochemical methods on cotton fabrics. Synth. Met. 2009, 159, 1353-1358. [CrossRef]

47. Zhang, B.; Xu, Y.; Zheng, Y.; Dai, L.; Zhang, M.; Yang, J.; Chen, Y.; Chen, X.; Zhou, J. A facile synthesis of polypyrrole/carbon nanotube composites with ultrathin, uniform and thickness-tunable polypyrrole shells. Nanoscale Res. Lett. 2011, 6, 2-9. [CrossRef] [PubMed]

48. Yaghoubidoust, F.; Wicaksono, D.H.B.; Chandren, S.; Nur, H. Effect of graphene oxide on the structural and electrochemical behavior of polypyrrole deposited on cotton fabric. J. Mol. Struct. 2014, 1075, 486-493. [CrossRef]

49. Xu, J.; Zhang, J.; Gao, W.; Liang, H.; Wang, H.; Li, J. Preparation of chitosan/PLA blend micro/nanofibers by electrospinning. Mater. Lett. 2009, 63, 658-660. [CrossRef]

50. Zhua, A.; Zhangb, M.; Wua, J.; Shen, J. Covalent immobilization of chitosan/heparin complex with a photosensitive hetero-bifunctional crosslinking reagent on PLA surface. Biomaterials 2002, 23, 4657-4665. [CrossRef]

51. Um, I.C.; Kweon, H.; Park, Y.H.; Hudson, S. Structural characteristics and properties of the regenerated silk fibroin prepared from formic acid. Biomaterials 2001, 29, 91-97. [CrossRef]

52. Belbachir, K.; Noreen, R.; Gouspillou, G.; Petibois, C. Collagen types analysis and differentiation by FTIR spectroscopy. Anal. Bioanal. Chem. 2009, 395, 829-837. [CrossRef] [PubMed]

53. Wu, J.; Li, Q.; Fan, L.; Lan, Z.; Li, P.; Lin, J.; Hao, S. High-performance polypyrrole nanoparticles counter electrode for dye-sensitized solar cells. J. Power Sources 2008, 181, 172-176. [CrossRef]

(C) 2018 by the authors. Licensee MDPI, Basel, Switzerland. This article is an open access article distributed under the terms and conditions of the Creative Commons Attribution (CC BY) license (http:/ / creativecommons.org/licenses/by/4.0/). 